\title{
Formulation of Weighted Goal Programming Using the Data Analysis Approach for Optimising Vehicle Acoustics Levels
}

\author{
Zulkifli Mohd Nopiah \\ Department of Mechanical and Materials Engineering, Universiti Kebangsaan Malaysia, 43000, Bangi, Selangor, \\ Malaysia
}

\author{
Ahmad Kadri Junoh \\ Institute of Engineering Mathematics, Universiti Malaysia Perlis, 026 00, Arau, Perlis, Malaysia
}

\begin{abstract}
Ahmad Kamal Ariffin
Department of Mechanical and Materials Engineering, Universiti Kebangsaan Malaysia, 43000, Bangi, Selangor, Malaysia
\end{abstract}

(Received 25 March 2012; revised 13 August 2012; accepted 14 November 2012)

Two factors for customers to consider when purchasing a vehicle are the vehicle acoustical comfort and the vibration in the vehicle cabin, both of which contribute to a comfortable driving environment. The amount of discomfort is included by frequency, magnitude, direction, and the period during which the noise is experienced and also where the vibration is experienced in the cabin. The main sources of vibration that have been identified previously are the vibration transmitted from engine and the vibration transmitted from the interaction of the tires with the road surface. In this study, we investigate the effect of the vibration caused by the tire interaction with the road surface by estimating the amount of noise produced due to this phenomenon. The methodology focuses on the trends which occur in the noise exposure and on the vibration exposure that has been generated throughout the engine operating rpm range in both stationary and non-stationary conditions. The vibration dose value (VDV)f was used to assess the amount of vibration exposure that is transmitted to the driver's body in the cabin. Through the study, we have proved that the vibration caused by the tire-road surface contact is a major contributor to the cabin's interior noise. Based on the results, a goal programming method was developed to optimise the noise levelin the cabin by considering the vibration as an input in the model. Finally, a multi-objective goal programming was developed successfully which could be used to optimize the noise level in the cabin by looking at the value of the VDV required at particular engine speeds (rpm).

\section{INTRODUCTION}

Vibration in vehicles produces noise that affects the emotions and focus of the drivers. The exposure to the vibration will affect visual performance by producing blurred visual images, since the vibration can cause relative movement between the retina and the objects viewed. This scenario can affect driving comfort in that it can reduce the focus of a driver and it has the potential to cause road accidents. Based on previous research which examined the vehicle cabin acoustical environment, the main sources of noise that have been identified may be categorized into a few main sources, namely the transmission of the engine, the exhaust exit, and the noise from the tires that is produced by the tire-road contact. However, in this study, we focused on the main source of the vibration caused by tire interaction with the road surface. ${ }^{1,2}$ Based on previous studies concerning vehicles moving at a constant rate of speed, the dominant source of noise was found to be the vibration caused by tire-road contact.

In order to evaluate the noise level in the cabin, various stan- dards and methodologies were considered and used, to predict the changes in sound quality while the vehicle was operated in both stationary and non-stationary conditions. ${ }^{3,4}$ The findings from the results can be used particularly by vehicle manufacturers to modify and improve the construction of vehicle to reduce the vibration exposure along with the reduction of noise in the cabin..$^{5,6}$

Various studies have been conducted by researchers to identify and estimate the generation of noise and vibration in the vehicle cabin in both stationary and non-stationary conditions. Generally, the transmission of noise into the vehicle is caused by two main sources: (1) airborne noise and (2) structure borne noise. One of the sources that contribute to the vehicle's interior noise is the vibration caused by the tire-road contact. With regard to this, some studies have been carried out to investigate the effect of the tire tread rubber on the noise generated. As a result, various characteristics of the tread rubber will produce different levels of noise that are caused by different levels of vibration.

Following the research carried out previously, it has been 